\title{
Persea americana Mill.: As a potent quorum sensing inhibitor of Pseudomonas aeruginosa PAO1 virulence
}

\author{
Fatma Tugce Guragac Dereli ${ }^{\circledR 1}$, Ebru Onem ${ }^{(2, *}$, Evren Arin ${ }^{(3)}$, Ayse Gul Ozaydin $\left(\mathbb{D}^{4}\right.$, \\ Muhammed Tilahun Muhammed 5
}

\begin{abstract}
${ }^{1}$ Süleyman Demirel University, Faculty of Pharmacy, Department of Pharmacognosy, Isparta, Turkiye ${ }^{2}$ Süleyman Demirel University, Faculty of Pharmacy, Department of Pharmac. Microbiology, Isparta, Turkiye ${ }^{3}$ Süleyman Demirel University, Vocational School of Health Services, Department of Anesthesia, Isparta, Turkey ${ }^{4}$ Suleyman Demirel University, YETEM-Innovative Technology Applic. and Research Center, Isparta, Turkiye ${ }^{5}$ Süleyman Demirel University, Faculty of Pharmacy, Department of Pharmaceutical Chemistry, Isparta, Turkiye
\end{abstract}

Abstract: The emergence of bacteria resistant to conventional antibiotics and the inability of these antibiotics to treat bacterial biofilm-induced infections cause millions of deaths every year.

This situation has prompted scientists to develop alternative strategies to combat infectious diseases. Among these, researches on phytochemicals to reduce bacterial virulence in Pseudomonas aeruginosa have gained momentum in recent years. The main reasons behind this are the production of virulence factors and biofilm formation, all of which are under the control of quorum sensing (QS) system. Hence, inhibition of the QS pathways is an eligible strategy for the control of microbial pathogenesis.

For the first time in the present study, the methanolic seed extract of avocado was evaluated for its anti-QS activity against $P$. aeruginosa PAO1. The results of the experiments carried out proved that the extract has inhibitory activity on the regulation of virulence and biofilm formation. Phytochemical analysis resulted in the identification of epicatechin, catechin, chlorogenic acid, caffeic acid, quercetin, kaempferol, vanillin, ferulic acid in the extract. Then, the mechanism of action for the extract was investigated through molecular docking. Docking outcomes demonstrated that the major components, catechin, epicatechin, chlorogenic acid, could bind to the receptors of QS competitively. Hence, the mode of action for the extract might be through the inhibition of the QS. Considering the computational analysis results and the literature, it is thought that the anti-QS activity of the extract prepared from avocado seeds may be related to the synergistic effect of the phytochemicals it contains.

\section{ARTICLE HISTORY}

Received: Nov. 28, 2021

Accepted: Jan. 18, 2022

\section{KEYWORDS}

Persea americana,

Biofilm,

PAO1,

Phytochemical,

Molecular docking.

\section{INTRODUCTION}

Antimicrobial resistance is defined as the capacity of microorganisms to develop various mechanisms that inactivate antimicrobial agents. As one of the most serious threats to global health, it causes millions of deaths and results in huge financial losses each year (Bery et al., 2013). Since the discovery of new conventional antibiotics targeting bacterial killing or

${ }^{*}$ CONTACT: Ebru Onem $\bigotimes$ ebruonem@sdu.edu.tr $\equiv$ Süleyman Demirel University, Faculty of Pharmacy, Department of Pharmaceutical Microbiology, Isparta, Turkiye 
inhibition to overcome the threat of resistance is not a permanent solution, this situation has prompted scientists to develop alternative strategies to combat infectious diseases. Among these, down-regulation of QS system associated with bacterial virulence is the most remarkable strategy in the last few decades (Jiang et al., 2019).

QSis a survival mechanism of bacteria and provides resistance against antimicrobial chemotherapeutics by controlling a variety of pathophysiological processes related to bacterial functions through cell-to-cell signaling (Rutherford \& Bassler, 2012). This population densitydependent intercellular communication network is formed by signal molecules called autoinducers (Smith \& Iglewski, 2003). The autoinducer concentration that reaches the critical threshold causes changes in the gene expression of the bacteria as a result of interaction with the QS receptors and triggers the regulation of a range of biochemical processes. In this fashion, bacteria gain the ability to adapt to environmental changes important for growth, adhesion, antibiotic resistance, and virulence (Parsek \& Greenberg, 1999). QS-associated biofilm formation and production of virulence factors reduce sensitivity to antibacterial therapy. In this regard, inhibition of the QS system is vital in combating life-threatening bacterial infections (Vysakh et al., 2018).

The opportunistic Gram-negative bacterium Pseudomonas aeruginosa is responsible for a broad spectrum of infectious diseases and is classified as a major cause of nosocomial infections (Lyczak et al., 2000). It has been listed by World Health Organization (WHO) as one of the 12 major pathogens of critical priority that are considered the greatest threat to human health (Tacconelli et al., 2018). Pseudomonal infections are correlated with high morbidity and mortality and are really difficult to treat due to the high resistance of bacteria to multiple classes of disinfecting agents. The main reasons behind this antimicrobial resistance are the production of virulence factors (like pyocyanin, rhamnolipids, exotoxins, proteases, elastases) and biofilm formation, all of which are under the control of the QS system (Pompilio et al., 2015). Hence, inhibition of the QS pathways is an eligible strategy for the control of microbial pathogenesis.

Medicinal plants that have inspired the discovery of new medicines have attracted great attention throughout the ages (Rather et al., 2021). Recently, there has been increased interest in studying the phytochemicals responsible for QS inhibition in the treatment of infections caused by resistant microorganisms (Mohabi et al., 2017).

Persea americana Mill. is one of two species belonging to the genus Persea (the other is $P$. schiedeana) and is the most studied member of this genus. It is an evergreen tree classified into the family Lauraceae and native to tropical America. Today, it is widely cultivated commercially for its edible fruit known as "avocado" in tropical and subtropical regions (Hurtado-Fernández et al., 2018). Avocado consumption has increased tremendously with increased awareness of its health benefits. It is considered as one of the healthiest fruits due to its rich nutritional composition, which includes vitamins, minerals, proteins and monounsaturated fatty acids (Dreher \& Davenport, 2013). Its unique phytochemical content has paved the way for this fruit to be researched for medicinal applications, and so far different parts of the fruit have been studied for their antioxidant, anticancer, anti-inflammatory, antimicrobial, antidiabetic, hypolipidemic, hepatoprotective, antihemolytic and wound healing activities (Nayak et al.,2008; Rodriguez-Carpena et al., 2011; Pahua-Ramos et al., 2012; Nabavi et al., 2013; Alkhalaf et al., 2019; Umoh et al., 2019). However, there is no literature data currently exists on the anti-QS activity of any parts of avocado.

In the present study described here, we aimed to evaluate the QS inhibitory activity of the methanolic seed extract of avocado against $P$. aeruginosa PAO1. In addition, the mechanism of action for QS inhibition detected was explored through computational analysis. 


\section{MATERIAL and METHODS}

\subsection{Plant Material and Extract Preparation}

Samples of matured avocado fruits were collected from the Manavgat region of Turkey. The herbarium sample was identified as $P$. americana Mill. by Asst. Prof. Gülsen Kendir and has been deposited with voucher number AEF 30121 in Herbarium of Ankara University Faculty of Pharmacy.

The seeds were removed from the succulent parts of the fruits by knife and washed with distilled water. After that, they were sliced and dried in an oven at $36{ }^{\circ} \mathrm{C}$ to a constant weight. They were then ground into powder using a grinding machine (Waring $8011 \mathrm{~EB}$ ). Eight grams of seed powder was subjected to ultrasonic extraction with $80 \mathrm{~mL}$ for $45 \mathrm{~min}$. The methanolic seed extract was filtered and the filtrate was evaporated to dryness at $36{ }^{\circ} \mathrm{C}$ using a rotary evaporator (Heidolph Hei-Vap Rotary Evaporator). At the end of the process, the crude extract remaining in the flask was weighed and the amount recorded, then dissolved with dimethylsulfoxide (DMSO) and transferred to a vial.

\subsection{Phytochemical Screening}

The phytochemical analysis of methanolic seed extract was carried out High-Performance Liquid Chromatography (HPLC) technique. HPLC conditions were presented in Table 1.

Table 1. Chromatographic conditions.

\begin{tabular}{lccc}
\hline Chromatographic conditions & $\begin{array}{c}\text { Time } \\
(\text { min.) }\end{array}$ & $\begin{array}{c}\text { A } \\
(\%)\end{array}$ & $\begin{array}{c}\text { B } \\
(\%)\end{array}$ \\
\hline Detector: & 0 & 93 & 7 \\
Photo Diode Array Detector $(\lambda$ max.: 278 nm) & 20 & 72 & 28 \\
Autosampler: & 28 & 75 & 25 \\
SIL-10AD vp & 35 & 70 & 30 \\
System controller: & 50 & 70 & 30 \\
SCL-10A vp & 60 & 67 & 33 \\
Pump: & 62 & 58 & 42 \\
LC-10AD vp & 70 & 50 & 50 \\
Degasser: & 73 & 30 & 70 \\
DGU-14a & 75 & 20 & 80 \\
Column heater: & 80 & 0 & 100 \\
CTO-10 A vp & & & \\
Column: & & & \\
Agilent Eclipse XDB C-18 & & & \\
$(250$ mm $\times 4.6$ mm), 5 $\mu$ m & & & \\
Column temperature: & 81 & 93 & \\
$30{ }^{\circ} \mathrm{C}$ & & & \\
Mobile phases: & & & \\
A: acetic acid-water $(3: 97$ v/v), B: methanol & & & \\
Flow rate: & & & \\
0.8 mL/min. & & & \\
Injection volume: & & & \\
$20 \mathrm{~L}$ & & & \\
\hline
\end{tabular}

\subsection{Screening Seed Extract for QS Inhibitory Activity}

\subsubsection{Antibacterial activity}

QS inhibitors should reduce virulence rather than bacterial growth, in contrast to standard antimicrobials. Therefore, firstly, the agar well method was used to determine the concentration with no antibacterial effect on PAO1 (Holder \& Boyce, 1994). Overnight cultures of bacteria were prepared by adjusting to $0.5 \mathrm{McFarland}$ turbidity. Five $\mathrm{mL}$ soft agar $(0.5 \%$ agar $)$ with 
bacterial cultures, added on the Muller-Hinton Agar (MHA) medium and $6 \mathrm{~mm}$ diameter wells were opened on the media. $100 \mu \mathrm{L}$ of the extract was added to the well. Antibacterial activity was determined by measuring the zone diameters after 24 hours of incubation at $35^{\circ} \mathrm{C}$. The test was carried out in triplicate.

\subsubsection{Biofilm formation assay}

Biofilm is a virulence trait associated with QS known to protect pathogens from host defense as well as antibiotics by acting as a diffusion barrier (Xu et al., 2000). Centers for Disease Control and Prevention (CDC) reported that approximately $65-80 \%$ of infections are caused by biofilm and this reveals the need for new treatment options to be developed in this regard $(\mathrm{Qu}$ et al., 2016).

The anti-biofilm activity of the seed extract was investigated on $P$. aeruginosa PAO1 strain using the crystal violet method (O'Toole 2011; Önem et al., 2018). $10 \mu \mathrm{L}$ of an overnight culture of PAO1 (OD at $600 \mathrm{~nm}=0,05$ ) was added to a 96-well microplate containing $160 \mu \mathrm{L}$ of freshly prepared Luria-Bertani Broth (LBB) medium and $20 \mu \mathrm{L}$ of the seed extract. Microplate incubated at $37^{\circ} \mathrm{C}$ for $48 \mathrm{~h}$. After the incubation, the culture on the plates was drained and washed 3 times with sterile water. By adding $125 \mu \mathrm{L}$ aqueous solution of crystal violet $(0.1 \%)$ to the wells, the biofilm layer was dyed for $30 \mathrm{~min}$, then the paint was poured and the excess was washed with distilled water. Two hundred $\mu \mathrm{L}$ of $95 \%$ ethanol was added and the reaction mixture was read spectrophotometrically at $570 \mathrm{~nm}$. PAO1 culture and LBB were used as positive and negative controls, respectively. All experiments were repeated three times unless otherwise mentioned. The inhibition that occurred in biofilm formation was calculated according to the following formula:

*OD: Optic Density

$$
\text { Inhibition rate }(\%)=\left[\left(\mathrm{OD}_{\text {in control }}-\mathrm{OD} \text { in treatment }\right) \times 100\right] / \mathrm{OD} \text { in control }
$$

\subsubsection{Elastolytic assay}

Elastase B also named LasB is an extracellular virulence factor of P. aeruginosa and this metalloprotease is involved in the invasiveness of this pathogen in the host tissues due to its ability to hydrolysis of immunologically important molecules such as antibodies (Bever \& Iglewski, 1988; Galdino et al., 2019).

The elastolytic activity of the seed extract was determined with Elastin Congo Red (ECR) test Ohman et al., 1988). This test helps to measure the elastase activity in the supernatant of PAO1 culture using ECR as substrate. Elastase B degrades elastin and this causes the congo red dye to be released into the supernatant. Elastolytic activity is determined by spectrophotometric quantification.

During the procedure, $100 \mu \mathrm{L}$ of the seed extract was mixed with $10 \mathrm{~mL}$ LBB containing OD 0.05 at $600 \mathrm{~nm}$ PAO1 culture and left to incubate at $37^{\circ} \mathrm{C}$ by shaking for $16-18 \mathrm{~h}$. Afterward, $100 \mu \mathrm{L}$ of the supernatant part of this culture was transferred to a tube and $900 \mu \mathrm{L}$ ECR buffer was added. This mixture was incubated at $37^{\circ} \mathrm{C}$ for $3 \mathrm{~h}$ with shaking at $200 \mathrm{rpm}$. After the incubation, the sample was centrifuged at $4500 \mathrm{rpm}$ for $5 \mathrm{~min}$. The supernatant of the sample was transferred to a cuvette and its optical absorption at $495 \mathrm{~nm}$ wavelength was measured spectrophotometrically (BioTek -Epoch 2 Microplate Spectrophotometer). The reference PAO1 strain was used as a positive control in this experiment. The negative control was sterile LBB.

\subsubsection{Pyocyanin inhibition assay}

Pyocyanin is a QS-controlled secondary metabolite exclusively produced by $P$. aeruginosa. Therefore, this redox-active toxic compound plays a role as an important biomarker in the identification of this pathogen (Reyes et al., 1988). As one of the virulence factors, it contributes 
to the persistence of pseudomonal infections. Reactive oxygen species associated with pyocyanin have been found to increase the survival ability of this opportunistic pathogen by helping to escape host defense, competing with other pathogens, and causing damage to the host tissue (Lau et al., 2004).

Pyocyanin inhibition assay was conducted as described by Essar et al., 1990. 10mL of LBB medium together with $100 \mu \mathrm{L}$ of plant extract left for incubation at $37^{\circ} \mathrm{C}$ for $16-18 \mathrm{~h}$ with shaking. After the incubation period, $5 \mathrm{~mL}$ of chloroform was added to the medium and vortexed for $30 \mathrm{sec}$. The sub-phase formed in the medium and separated from chloroform was transferred to tubes as $2 \mathrm{~mL}$. One $\mathrm{mL} \mathrm{HCl}$-water mixture $(0.2 \mathrm{~mol} / \mathrm{L} \mathrm{HCl})$ was added to it and vortexed for $30 \mathrm{sec}$ again. The absorbance of the pink phase formed on the upper part of the tubes was measured at $520 \mathrm{~nm}$. Untreated PAO1 was served as a positive control.

\subsection{Statistical Analysis}

The experiments were carried out in triplicate according to the randomized plot design and the data obtained were subjected to variance analysis using the JMP 8 packet statistics program. Statistical differences were marked by the LSD multiple comparison test.

\subsection{Molecular Docking}

The crystal structure of LasR was obtained from PDB (protein data bank). The structure utilized in the molecular docking (PDB ID: 6MWL) has a resolution of $1.50 \AA$ (Paczkowski et al., 2019). GRID box was specified in a manner that included the bound ligand inside the protein structure. The protein structures were prepared by deleting water molecules, adding polar hydrogens, and assigning Gasteiger charges. The structures of the ligands analyzed were obtained from PubChem (Kim et al., 2021). Similarly, the ligands were prepared for docking by adding polar hydrogens and assigning Gasteiger charges. Then, AutoDock Vina was run after the parameters were assigned properly (Trott \& Olson, 2010). The results were visualized and analyzed with Biovia Discovery Studio 3.5 (2020). The docking process was validated by performing redocking with the bound ligand in the structures utilized.

\section{RESULTS}

\subsection{Confirmation of the anti-QS activity}

Before the anti-quorum sensing experiments, an antibacterial activity test was performed to determine the concentration where the plant extract did not have antibacterial activity and it was observed that there was no activity up to $238 \mathrm{mg}$.

The results of the antibiofilm formation assay are given in Figure 1. The extract inhibited biofilm formation of PAO1 by $38 \%(2.38 \mathrm{mg} / \mathrm{mL}$ concentration).

Figure 1. Biofilm formation inhibition of plant seed extract.

${ }^{* *}$ The difference between averages with different letters is important, $p<0.01(S D \pm)$

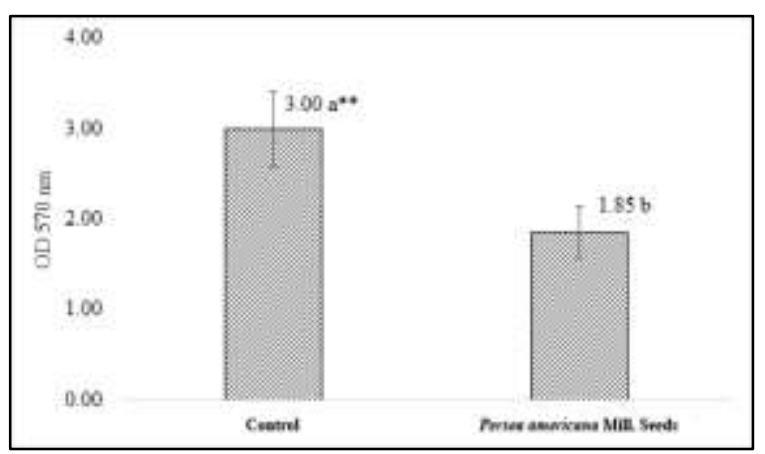

Figure 2. Elastase inhibition activity of plant seed extract.

${ }^{* *}$ The difference between averages with different letters is important, $p<0.01$ ( $S D \pm$ )

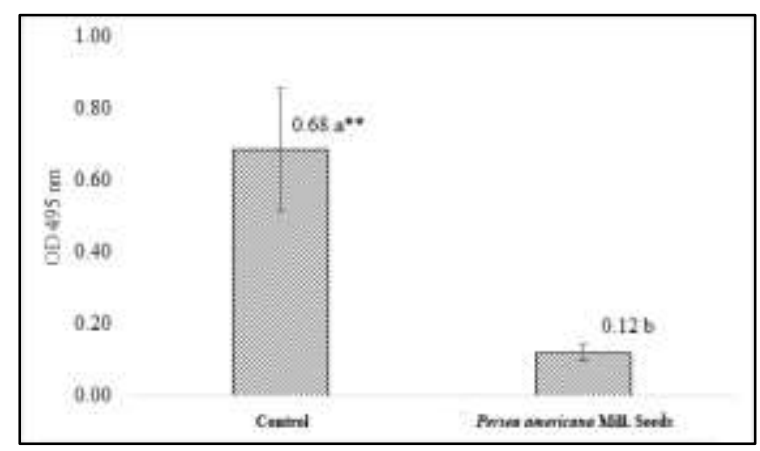


Results of the elastolytic assay are shown in Figure 2. Elastase inhibition rate of the extract was found as $83 \%$. Figure 3 presents the results of the pyocyanin inhibition assay. The percentage of pyocyanin inhibition of the extract was calculated as $79 \%$.

Figure 3. Pyocyanin inhibiton activity of plant seed extract.

${ }^{* *}$ The difference between averages with different letters is important, $p<0.01(S D \pm)$

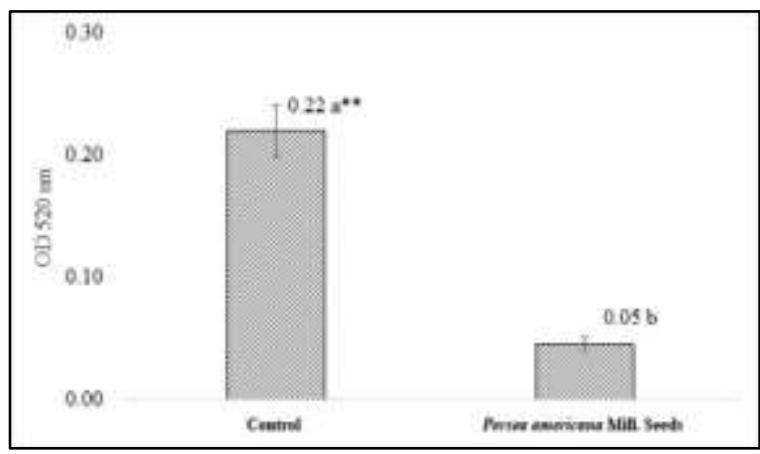

\subsection{Results of HPLC Analysis}

HPLC chromatogram of the methanolic extract of $P$. americana seeds shows the presence of gallic, $p$-hydroxybenzoic, chlorogenic, caffeic, ferulic acids and, catechin hydrate, epicatechin, vanillin, quercetin dihydrate, kaempferol. Figures $4 \mathrm{a} \& 4 \mathrm{~b}$ show a standards chromatogram and a sample chromatogram, respectively. Epicatechin had the highest concentration $(222.15$ $\mu \mathrm{g} / \mathrm{mL}$ ) followed by catechin with a concentration of $209.95 \mu \mathrm{g} / \mathrm{mL}$, then chlorogenic acid with a concentration of $97.65 \mu \mathrm{g} / \mathrm{mL}$, with the presence of $p$-hydroxybenzoic acid, caffeic acid, quercetin, kaempferol, vanillin, ferulic acid and gallic acid with concentrations of 82.2, 33.45, $21,8.75,6.25,4.45$ and $3.35 \mu \mathrm{g} / \mathrm{mL}$, respectively (Table 2). Chemical structures of determined compounds in the methanolic extract of avocado seeds are shown in Figure 5.

Figure 4. A) HPLC chromatogram for standards, B) HPLC chromatogram for the main phenolic compounds identified in the methanolic extract of $P$. americana Mill. seeds. 1:gallic acid; 2:protocatechic acid; 3:catechin; 4:p-hydroxybenzoic acid; 5:chlorogenic acid; 6:caffeic acid; 7:epicatechin; 8:syringic acid ; 9:vanillin; 10:p-coumaric acid; 11:ferulic acid; 12:sinapinic acid; 13:benzoic acid; 14:o-coumaric acid; 15:rutin; 16:hesperidin; 17:rosmarinic acid; 18:eriodictiol; 19:cinnamic acid; 20:quercetin; 21:luteolin; 22: kaempferol; 23:apigenin

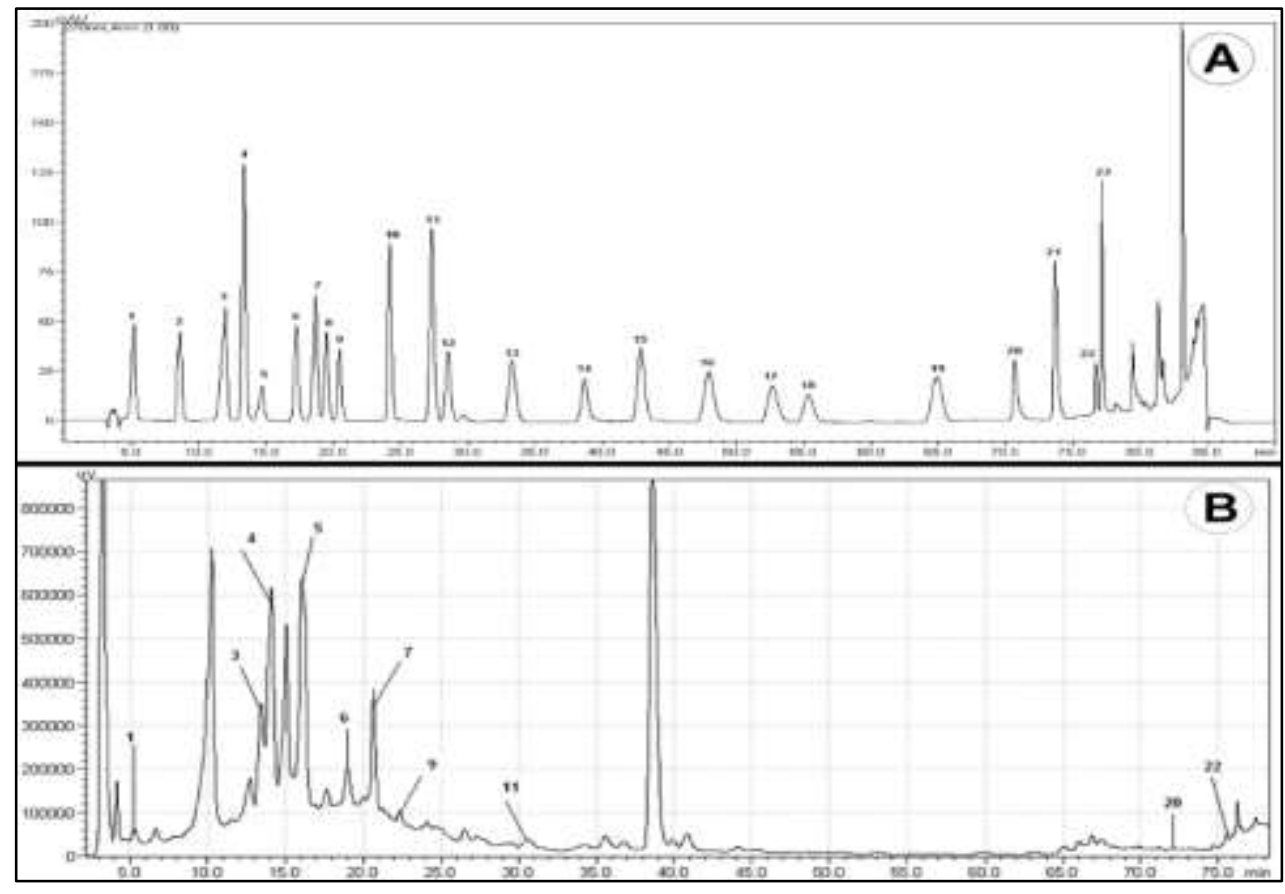


Table 2. Concentrations of the main phenolic compounds identified in the methanolic extract of $P$. americana Mill. seeds.

\begin{tabular}{lll}
\hline Phytochemicals & Concentrations $(\mu \mathrm{g} / \mathrm{mL})$ & Retention time $(\mathrm{min})$ \\
\hline Gallic acid & 3.35 & 5.41 \\
\hline Catechin & 209.95 & 13.50 \\
\hline p-Hydroxybenzoic acid & 82.2 & 14.60 \\
\hline Chlorogenic acid & 97.65 & 16.17 \\
\hline Caffeic acid & 33.45 & 18.84 \\
\hline Epicatechin & 222.15 & 20.57 \\
\hline Vanillin & 6.25 & 22.23 \\
\hline Ferulic acid & 4.45 & 30.23 \\
\hline Quercetin & 21 & 72.94 \\
\hline Kaempferol & 8.75 & 77.13 \\
\hline
\end{tabular}

Figure 5. Chemical structures of determined compounds in the methanolic extract of $P$. americana Mill. seeds. A) Epicatechin, B) Catechin, C) Chlorogenic acid, D) $p$-hydroxybenzoic acid, E) Caffeic acid, F) Quercetin, G) Kaempferol, H) Vanillin, I) Ferulic acid, J) Gallic acid.

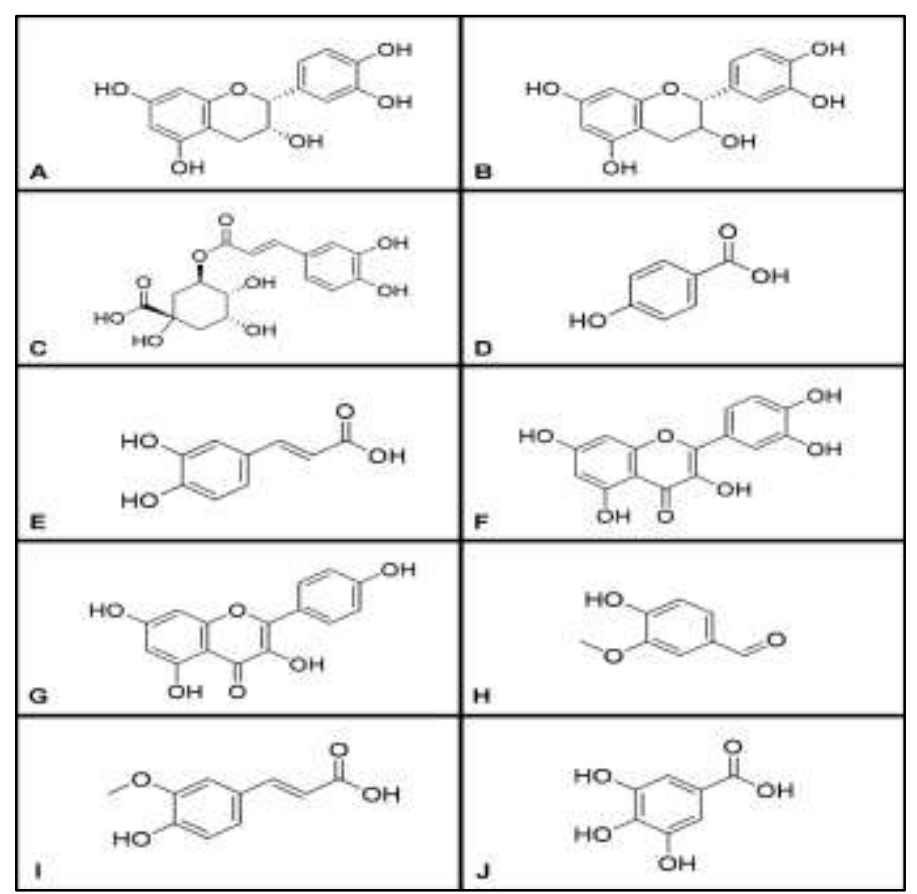

\subsection{Results of Molecular Docking Studies}

Molecular docking outcomes showed that catechin and its isomer epicatechin had relatively good interaction with LasR, a crucial receptor involved in the QS system (Figure 6). Hence, the ligands analyzed could bind to LasR very well. The binding energy of catechin, bound ligand and OdDHL (N-3-Oxo-Dodecanoyl-L-Homoserine Lactone) were recorded as $-11.9 \mathrm{kcal} / \mathrm{mol}$, $-10.5 \mathrm{kcal} / \mathrm{mol}$ and $-9.0 \mathrm{kcal} / \mathrm{mol}$ respectively. Furthermore, the interactions of catechin and epicatechin in the presence of water in the structure of the protein were investigated. In the presence of water molecules, the detected interactions were the same as the interactions without water. The only difference was the interaction with W438 (Figure 6 A\&B) (Lie et al., 2011). 
Figure 6. Binding mode of A) catechin B) catechin in hydrated protein structure C) bound ligand D) OdDHL with LasR. E) superimposition of the ligands inside the structure, F) ligands inside the binding pocket of LasR (green-epicatechin, yellow-bound ligand, magenta-OdDHL)

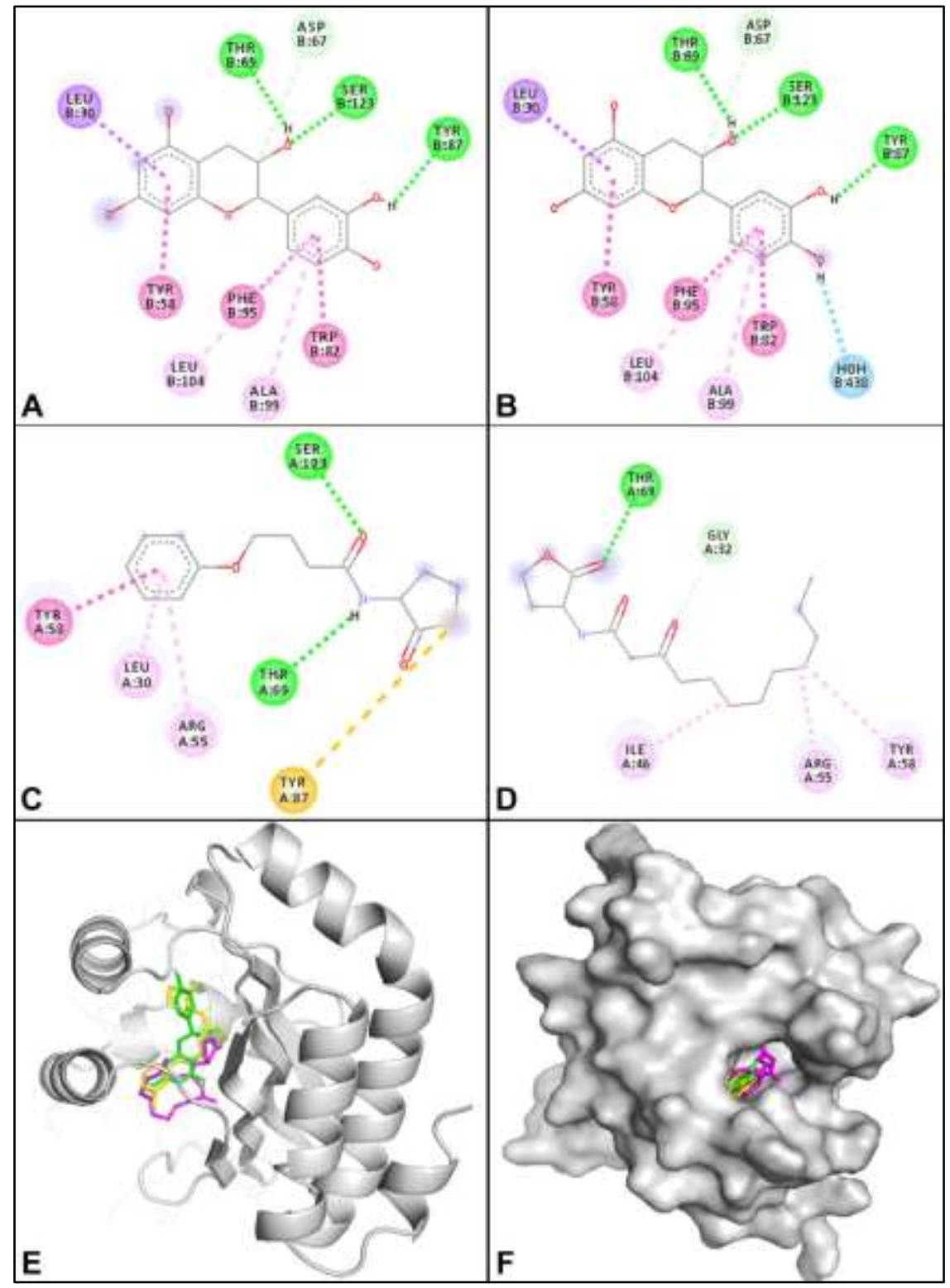

\section{DISCUSSION and CONCLUSION}

The emergence of bacteria resistant to conventional antibiotics and the inability of these antibiotics to treat infections caused by bacterial biofilms prove the need for new strategies in the treatment of bacterial infections (Saleem et al., 2010). Recently, researches on phytochemicals to reduce bacterial virulence in P. aeruginosa has gained momentum.

For the first time in the present study, the seed extract of avocado was evaluated for its antiQS activity against $P$. aeruginosa PAO1. Since methanol can extract a variety of bioactive phytochemicals better than the others, it was used as a solvent for the extraction of secondary metabolites. The results of the experiments carried out proved that the methanolic seed extract has inhibitory activity on the regulation of virulence and biofilm formation. Phytochemical analysis performed on the extract resulted in the identification of epicatechin, catechin, chlorogenic acid, $p$-hydroxybenzoic acid, caffeic acid, quercetin, kaempferol, vanillin, ferulic acid, and gallic acid. 
There are some data in the literature regarding the anti-QS activity of detected bioactive phytochemicals and the promising anti-QS and anti-biofilm activity of the extract has been associated with the synergistic effect of these phenolic compounds in its composition. Phenolic plant secondary metabolites are among the most investigated naturally occurring phytochemicals due to their health-promoting benefits (Bhuyan and Basu, 2017). These phytocompounds have attracted scientific interest in terms of their various biological activities, especially their antioxidant properties (Lattanzio et al., 2018). Additionally, some studies in recent years have provided evidence of the anti-QS and anti-biofilm activities of the phenolic phytoconstituents (Ugurlu et al., 2016). Flavonoidal compounds have been documented to interfere with the regulation of QS-associated pathways in PAO1. In the study by Vandeputte and associates, it was found that catechin has inhibitory activity on elastase and pyocyanin production and biofilm formation by downregulating QS gene expression in PAO1. On the other hand, it was determined that epicatechin also had an inhibitory effect on pyocyanin production (Vandeputte et al., 2010). Lahiri and colleagues have indicated that the catechin from Azadirachta indica leaf extract is extremely active in preventing dental biofilm and this compound can be used in the treatment of biofilm-related chronic infections (Lahiri et al., 2021). Quercetin, a flavonoid commonly found in the plant kingdom, has gained importance as a QS system inhibitor. Quyang et al. reported the inhibitory activity of this compound on virulence factors production and biofilm formation in PAO1 (Ouyang et al., 2021). The antiQS property of kaempferol, another flavonoidal compound, has been proven in a study investigating the effectiveness of phytochemicals obtained from Camellia nitidissima Chi flowers on PAO1 (Yang et al., 2018). Different studies showed the inhibitory potential of cinnamic acid derivatives against QS-controlled behaviours in PAO1. Wang and coworkers proved that chlorogenic acid regulated QS system and reduces the pathogenicity of $P$. aeruginosa by weakening virulence factors (Wang et al., 2019). In a study that investigated the effects of some phenolic secondary metabolites on QS-related virulence factor production of PAO1, caffeic and ferulic acids have been found to be active. The action mechanisms of these phenolic acids have been shown to be the reduction of pyocyanin production and blockage of biofilm formation (Ugurlu et al., 2016). Various studies have suggested that several benzoic acid derivatives present anti-QS activity against PAO1. In a study conducted by Plyuta et al., it was determined that gallic acid at a concentration of $200 \mu \mathrm{g} / \mathrm{mL}$ inhibits the formation of PAO1 biofilms by $30 \%$. In the same study, $p$-hydroxybenzoic acid and vanillin have been found to inhibit bacterial biofilm formation by reducing the swarming motility of the PAO1 strain in the concentration range of $400-800 \mu \mathrm{g} / \mathrm{mL}$ (Plyuta et al., 2013).

Phytochemical analysis of avocado extract revealed that catechin, epicatechin and chlorogenic acid are the first three most abundant components. Catechin and epicatechin were found to be the major components of the extract. In this study, the postulation was the QS inhibition effect detected might result from the inhibition of the QS receptors by these components synergistically. This premise was explored through molecular docking. Molecular docking outcomes demonstrated that catechin and epicatechin could inhibit the QS system by inhibiting LasR competitively. They bound to the ligand-binding domain of LasR with hydrogen bonding (Thr69, Tyr87, Ser123) and eight more hydrophobic interactions. The binding was realised at relatively low binding energy. In addition, the major components and the natural ligand (OdDHL) had common interaction points at Thr69 and Tyr58. According to the computational analysis here, catechin and epicatechin are expected to have stronger interaction with the LasR than the natural ligand (Figure 1). This in turn gives them the opportunity to inhibit the QS system by interfering with the binding of agonists to the receptor (Bottomley et al., 2007). Furthermore, previous experimental studies, which were supported by computational analysis, reported that chlorogenic acid had QS inhibition effect (Wang et al., 2019; Onem et al., 2021). 
To sum up, catechin, epicatechin and chlorogenic acid have the potential of inhibiting the QS system. Hence, the avocado extract, which consists of these compounds as major components, might inhibit this system and thus decrease bacterial virulence.

In conclusion, considering the computational analysis outcomes and literature data it is thought that the anti-QS activity of the methanolic extract prepared from avocado seeds may be due to the synergistic effect of phenolic phytochemicals in its content. Further studies are planned to undertaken to determine the anti-QS activity of different doses of isolated compounds thought to be responsible for the activity.

\section{Declaration of Conflicting Interests and Ethics}

The authors declare no conflict of interest. This research study complies with research and publishing ethics. The scientific and legal responsibility for manuscripts published in IJSM belongs to the authors.

\section{Authorship contribution statement}

Ebru Onem: Investigation, Supervision; Fatma Tugce Guragac Dereli: Writing-original draft; Ayse Gul Ozaydin: Methodology; Evren Arin: Resources, Methodology; Muhammed Tilahun Muhammed: Formal Analysis

\section{Orcid}

Fatma Tugce Guragac Dereli (D) https://orcid.org/0000-0002-7554-733X

Ebru Onem (i) https://orcid.org/0000-0002-7770-7958

Evren Arin (iD https://orcid.org/0000-0002-6800-9226

Ayse Gul Ozaydin (iD https://orcid.org/0000-0003-0050-5271

Muhammed Tilahun Muhammed (iD https://orcid.org/0000-0003-0050-5271

\section{REFERENCES}

Berry, D.B., Lu, D., Geva, M., Watts, J.C., Bhardwaj, S., Oehler, A., et al. (2013). Drug resistance confounding prion therapeutics. PNAS, 110(44), E4160-9. https://doi.org/10.107 $\underline{3 / \text { pnas. } 1317164110}$

Jiang, Q., Chen, J., Yang, C., Yin, Y., Yao, K. (2019). Quorum sensing: A prospective therapeutic target for bacterial diseases. BioMed Res. Int. 2015978. https://doi.org/10.1155/ 2019/2015978

Rutherford, S.T., Bassler, B.L. (2012). Bacterial quorum sensing: Its role in virulence and possibilities for its control. Cold Spring Harbor Perspect. Med., 2(11), a012427. https://doi.org/10.1101/cshperspect.a012427

Smith, R.S., Iglewski, B.H. (2003). P. aeruginosa quorum-sensing systems and virulence. Curr. Opin. Microbiol. 6(1), 56-60. https://doi.org/10.1016/S1369-5274(03)00008-0

Parsek, M.R., Greenberg, E.P. (1999). Quorum sensing signals in development of Pseudomonas aeruginosa biofilms. Meth. Enzymol., 310, 43-55. https://doi.org/10.1016/S00766879(99)10005-3

Vysakh, A., Midhun, S.J., Jayesh, K., Jyothis, M., Latha, M.S. (2018). Studies on biofilm formation and virulence factors associated with uropathogenic Escherichia coli isolated from patient with acute pyelonephritis. Pathophysiology., 25(4), 381-7. https://doi.org/10.1016/j. pathophys.2018.07.004

Lyczak, J.B., Cannon, C.L, Pier, G.B. (2000). Establishment of Pseudomonas aeruginosa infection: Lessons from a versatile opportunist. Microbes and Infect., 2(9), 1051-60. https://doi.org/10.1016/S1286-4579(00)01259-4

Tacconelli, E., Carrara, E., Savoldi, A., Harbarth, S., Mendelson, M., Monnet, D.L., et al. (2018). Discovery, research, and development of new antibiotics: The WHO priority list of 
antibiotic-resistant bacteria and tuberculosis. The Lancet Infect. Dis., 18(3), 318-27. https://doi.org/10.1016/S1473-3099(17)30753-3

Pompilio, A., Crocetta, V., Nicola, D.S., Verginelli, F., Fiscarelli, E., Bonaventura G.D. (2015). Cooperative pathogenicity in cystic fibrosis: Stenotrophomonas maltophilia modulates Pseudomonas aeruginosa virulence in mixed biofilm. Front. Microbiol., 6, 951. https://doi.org/10.3389/fmicb.2015.00951

Rather, M.A., Gupta, K., Mandal, M. (2021). Inhibition of biofilm and quorum sensingregulated virulence factors in Pseudomonas aeruginosa by Cuphea carthagenensis (Jacq.) J. F. Macbr. leaf extract: An in vitro study. J. Ethnopharmacol., 269, 113699. https://doi.org/10.1016/j.jep.2020.113699

Mohabi, S., Kalantar-Neyestanaki, D., Mansouri, S. (2017). Inhibition of quorum sensingcontrolled virulence factor production in Pseudomonas aeruginosa by Quercus infectoria gall extracts. Iran. J. Microbiol., 9(1), 26-32. PMID: 28775820 ; PMCID: PMC5534001.

Hurtado-Fernández, E., Fernández-Gutiérrez, A., Carrasco-Pancorbo, A. (2018). Avocado fruit-Persea americana. Academic Press.

Dreher, M.L., Davenport, A.J. (2013). Hass avocado composition and potential health effects. Crit Rev Food Sci Nutr., 53(7), 738-50. https://doi.org/10.1080/10408398.2011.556759

Nayak, B.S., Raju, S.S., Chalapathi, R.A.V. (2008). Wound healing activity of Persea americana (avocado) fruit: A preclinical study on rats. J. Wound Care., 17(3), 123-6. https://doi.org/10.12968/jowc.2008.17.3.28670

Rodriguez-Carpena, J.G., Morcuende, D., Andrade, MJ., Kylli, P., Estevez, M. (2011). Avocado (Persea americana Mill.) phenolics, in vitro antioxidant and antimicrobial activities, and inhibition of lipid and protein oxidation in porcine patties. J. Agric. Food Chem., 59(10), 5625-35. https://doi.org/10.1021/jf1048832

Pahua-Ramos, M.E, Ortiz-Moreno, A., Chamorro-Cevallos, G., Hernandez-Navarro, M.D., Garduno-Siciliano, L., Necoechea-Mondragon, H., et al. (2012). Hypolipidemic effect of avocado (Persea americana Mill) seed in a hypercholesterolemic mouse model. Plant Foods Hum. Nutr., 67(1), 10-16. https://doi.org/10.1007/s11130-012-0280-6

Nabavi, S.F., Nabavi, S.M, Setzer, W.N, Nabavi, S.A, Nabavi, S.A, Ebrahimzadeh, M.A. (2013). Antioxidant and antihemolytic activity of lipid-soluble bioactive substances in avocado fruits. Fruits, 68(3), 185-93. https://doi.org/10.3390/antiox8100426

Alkhalaf, M.I, Alansari, W.S, Ibrahim, E.A, ELhalwagy, M.E.A. (2019). Anti-oxidant, antiinflammatory and anti-cancer activities of avocado (Persea americana) fruit and seed extract. Journal of King Saud University-Science, 31(4), 1358-62. https://doi.org/10.3389/f nut.2021.775751

Umoh, I.O., Samuel, O.O., Kureh, T.B., Davies, K.G. (2019). Antidiabetic and hypolipidaemic potentials of ethanol fruit pulp extract of Persea americana (avocado pear) in rats. J. Afr. Assoc. Physiol., 7(1), 59-63.

Holder, I.A., Boyce, S.T. (1994). Agar well diffusion assay testing of bacterial susceptibility to various antimicrobials in concentrations non-toxic for human cells in culture. Burns, 20(5), 426-29. https://doi.org/10.1016/0305-4179(94)90035-3

$\mathrm{Xu}$, K.D., McFeters, G.A., Stewart, P.S. (2000). Biofilm resistance to antimicrobial agents. Microbiology, 146, 547-49. https://doi.org/10.1099/00221287-146-3-547

Qu, Y., Locock, K., Verma-Gaur, J., Hay, I.D., Meagher, L., Traven, A. (2016). Searching for new strategies against polymicrobial biofilm infections: Guanylated polymethacrylates kill mixed fungal/bacterial biofilms. J. Antimicrob. Chemother., 71(2), 413-21. https://doi.org/ $10.1093 / \mathrm{jac} / \mathrm{dkv} 334$

O'Toole, G.A. (2011). Microtiter dish biofilm formation assay. Journal of Visualized Experiments, 30(47), 2437. https://doi.org/10.3791/2437 
Onem, E., Dundar, Y., Ulusoy, S., Noyanalpan, N., Bosgelmez-Tinaz, G. (2018). Anti-quorum sensing activity of 1, 3-dihydro-2H-benzimidazol-2-one derivatives. Fresenius Environ. Bull., 27(12 B), 9906-12.

Bever, R.A., Iglewski, B.H. (1988). Molecular characterization and nucleotide sequence of the Pseudomonas aeruginosa elastase structural gene. J. Bacteriol., 170(9), 4309-14. https://doi .org/10.1128/jb.170.9.4309-4314.1988

Galdino, A.C.M., de Oliveira, M.P., Ramalho, T.C., de Castro, A.A., Branquinha, M.H., Santos, A.L.S. (2019). Anti-virulence strategy against the multidrug-resistant bacterial pathogen Pseudomonas aeruginosa: Pseudolysin (Elastase B) as a potential druggable target. Curr. Protein Pept. Sci., 20(5), 471-87. https://doi.org/10.2174/1389203720666190207100415

Ohman, D.E., Cryz, S.J., Iglewski, B.H. (1980). Isolation and characterization of Pseudomonas aeruginosa PAO mutant that produces altered elastase. J. Bacteriol., 142(3), 836-42. https://doi.org/10.1128/jb.142.3.836-842.1980

Reyes, E.A., Bale, M.J., Cannon, W.H., Matsen, J.M. (1981). Identification of Pseudomonas aeruginosa by pyocyanin production on Tech agar. J. Clinic. Microbiol., 13(3), 456-8. https://doi.org/10.1128/jcm.13.3.456-458.1981

Lau, G.W., Hassett, D.J., Ran, H., Kong, F. (2004). The role of pyocyanin in Pseudomonas aeruginosa infection. Trends. Mol. Med., 10(12), 599-606. https://doi.org/10.1016/j.molme d.2004.10.002

Essar, D.W., Eberly, L., Hadero, A., Crawford, I.P. (1990). Identification and characterization of genes for a second anthranilate synthase in Pseudomonas aeruginosa: Interchangeability of the two anthranilate synthases and evolutionary implications, J. Bacteriol., 172(2), 884900. https://doi.org/10.1128/jb.172.2.884-900.1990

Paczkowski, J.E., McCready, A.R., Cong, J.P., Li, Z., Jeffrey, P.D., Smith, C.D., et al. (2019). An autoinducer analog reveals an alternative mode of ligand binding for the LasR quorum-sensing receptor. ACS Chem. Biol., 14(3), 378-89. https://doi.org/10.1021/acschem bio. $8 \mathrm{~b} 00971$

Kim, S., Chen, J., Cheng, T., Gindulyte, A., He, J., He, S., et al. (2021). PubChem in 2021: New data content and improved web interfaces. Nucleic Acids Res., 49(D1), D1388-95. https://doi.org/10.1093/nar/gkaa971

Trott, O., Olson, A.J. (2010). AutoDock Vina: Improving the speed and accuracy of docking with a new scoring function, efficient optimization, and multithreading. J. Comput. Chem., 31(2), 455-61. https://doi.org/10.1002/jcc.21334

Lie, M.A., Thomsen, R., Pedersen, C.N, Schiott, B., Christensen, M.H. (2011). Molecular docking with ligand attached water molecules. J Chem Inf Model., 51(4), 909-17. https://doi.org/10.1021/ci100510m

Saleem, M., Nazir, M., Ali, M.S., Hussain, H., Lee, Y.S., Riaz, N., et al. (2010). Antimicrobial natural products: An update on future antibiotic drug candidates. Nat. Pro. Rep., 27(2), 23854. https://doi.org/10.1039/B916096E

Bhuyan, D.J., Basu, A. (Eds). (2017). Phenolic compounds: Potential health benefits and toxicity. Western Sydney University.

Lattanzio, V., Kroon, P.A., Quideau, S., Treutter, D. (2008). Plant phenolics-Secondary metabolites with diverse functions. Wiley-Blackwell.

Ugurlu, A., Yagci, A.K., Ulusoy, S., Aksu, B., Bosgelmez-Tinaz, G. (2016). Phenolic compounds affect production of pyocyanin, swarming motility and biofilm formation of Pseudomonas aeruginosa. Asian Pac. J. Trop. Biomed., 6(8), 698-701. https://doi.org/10.10 16/j.apjtb.2016.06.008

Vandeputte, O.M., Kiendrebeogo, M., Rajaonson, S., Diallo, B., Mol, A., El Jaziri, M., et al. (2010). Identification of catechin as one of the flavonoids from Combretum albiflorum bark extract that reduces the production of quorum-sensing-controlled virulence factors in 
Pseudomonas aeruginosa PAO1. Appl. Environ. Microbiol., 76(1), 243-53. https://doi.org/1 0.1128/AEM.01059-09

Lahiri, D., Nag, M., Dutta, B., Mukherjee, I., Ghosh, S., Dey, A., et al. (2021). Catechin as the most efficient bioactive compound from Azadirachta indica with antibiofilm and antiquorum sensing activities against dental biofilm: An in vitro and in silico study. Appl. Biochem. Biotechnol., 1-14. https://doi.org/10.1007/s12010-021-03511-1

Ouyang, J., Sun, F., Feng, W., Sun, Y., Qiu, X., Xiong, L., et al. (2016). Quercetin is an effective inhibitor of quorum sensing, biofilm formation and virulence factors in Pseudomonas aeruginosa. Appl. Environ. Microbiol., 120(4), 966-74. https://doi.org/10.1111/jam.13073

Yang, R., Guan, Y., Zhou, J.W., Sun, B., Wang, Z.N., Chen, H.J., et al. (2018). Phytochemicals from Camellia nitidissima Chi flowers reduce the pyocyanin production and motility of Pseudomonas aeruginosa PAO1. Front. Microbiol., 8, 1-13. https://doi.org/10.3389/fmicb. 2017.02640

Wang, H., Chu, W.H., Ye, C., Gaeta, B., Tao, H.M., Wang, M., et al. (2019). Chlorogenic acid attenuates virulence factors and pathogenicity of Pseudomonas aeruginosa by regulating quorum sensing. Appl. Microbiol. Biotechnol., 103(2), 903-15. https://doi.org/10.1007/s00 253-018-9482-7

Plyuta, V., Zaitseva, J., Lobakova, E., Zagoskina, N., Kuznetsov, A., Khmel, I. (2013). Effect of plant phenolic compounds on biofilm formation by Pseudomonas aeruginosa. Apmis, 121(11), 1073-81. https://doi.org/10.1111/apm.12083

Bottomley, M.J., Muraglia, E., Bazzo, R., Carfî, A. (2007). Molecular insights into quorum sensing in the human pathogen Pseudomonas aeruginosa from the structure of the virulence regulator LasR bound to its autoinducer. J. Biol. Chem., 282, 13592-600. https://doi.org/10. 1074/jbc.M700556200

Onem, E., Sarisu, H.C., Ozaydin, A.G., Muhammed, M.T., Ak, A. (2021). Phytochemical profile, antimicrobial and anti-quorum sensing properties of fruit stalks of Prunus avium L. Lett. Appl. Microbiol., 73(4), 426-437. https://doi.org/10.1111/lam.13528 